\title{
From weight management via diabetes control to cardiovascular risk reduction
}

\begin{abstract}
Introduction: Since 1997, the author has been diagnosed with obesity, type 2 diabetes (T2D), hypertension, hyperlipidemia, and suffered five cardiac episodes. He spent 20,000 hours since 2010 to study and research his chronic diseases in order to save his own life. This abstract tells his story.
\end{abstract}

Method: He created a math-physical medicine approach, instead of using the traditional biochemical method, to conduct his research. Initially, he defined interrelationships among 11 categories and 500 elements of a human metabolism system. $\mathrm{He}$ collected and processed 1.5 million data of his lifestyle details and medical conditions. Furthermore, utilizing physics, mathematics, engineering modeling, and artificial intelligence (AI), he developed four prediction models with $99 \%$ accuracy, including weight, fasting plasma glucose, post prandial glucose, and hemoglobin A1C. Finally, he developed a risk probability calculation model of having heart attack or stroke.

Results: From the period of 2013-2018, he has reduced his weight from $220 \mathrm{lbs}$. to 167 lbs., waistline from 44" to 32", and BMI from 33.1 (obese) to 24.7 (normal). Based on his acquired knowledge, he developed AI-based prediction tools to reduce his average glucose value from $279 \mathrm{mg} / \mathrm{dL}$ to $116 \mathrm{mg} / \mathrm{dL}$, A1C from $10 \%$ to $6.5 \%$. Since 2016, his hypertension and hyperlipidemia are no longer health concerns along with dropping his cardiovascular risk from $74 \%$ to $31 \%$.

Conclusion: Over eight years, the author was finally able to control his weight and T2D along with greatly reducing his cardiovascular risk. In addition to his willpower and persistence, his diligence in acquiring medical knowledge from reading hundreds of textbooks and medical papers has assisted him. More importantly, his knowledge from other disciplines in mathematics, physics, engineering, statistics, computer science, and technology have provided him the necessary tools.
Volume 8 Issue 4 - 2018

\section{Gerald C Hsu}

EclaireMD Foundation, USA

Correspondence: Gerald C Hsu, 7 Oak Haven Way Woodside, CA 94062, EclaireMD Foundation, USA, Tel + |-5 | 0-33 |-5000, Email g.hsu@eclairemd.com

Received: July 24, 2018 | Published: August 02, 2018

\section{Introduction}

Since 1997, the author has been diagnosed with three chronic diseases such as type 2 diabetes (T2D), hypertension, and hyperlipidemia. As shown in Figure 1, his health reached to a "near collapsed" condition by 2010 ; therefore, he launched his type 2 diabetes (T2D) research in order to save his own life. From 2012 to 2018 , he has collected and processed approximately $1.5 \mathrm{M}$ data regarding his own health and body conditions. During 2010-2013, he studied six chronic diseases and food nutrition in depth. In 2014, using mathematics and various engineering modeling, he developed a mathematical model of the human metabolism system, which contains 11 categories and $\sim 500$ elements. Currently, as shown in Figure 1, his chronic disease conditions are now under control. This paper described his effort for 8-years in terms of annualized segments with different working methods utilized at different stages. It specifically discusses the relationship and results from weight management via diabetes control to lowering his risk probabilities of having heart attack or stroke.

\begin{tabular}{|c|c|c|}
\hline Health Exam Record & 2010 & 2017 \\
\hline $\operatorname{A1C}(<6.4 \%)$ & 10.0 & 6.1 \\
\hline $\begin{array}{l}\text { 90-days Average Glucose ( }<120 \mathrm{mg} / \\
\text { dL) }\end{array}$ & 279 & 113 \\
\hline $\operatorname{ACR}(<30)$ & \multicolumn{2}{|c|}{\begin{tabular}{|l|l|}
116.4 & 12.3 \\
\end{tabular}} \\
\hline Triglyceride $(<150)$ & 1161 & 67 \\
\hline HDL $(>40)$ & 24 & 48 \\
\hline LDL $(<130)$ & 174 & 74 \\
\hline Total Cholesterol $(<\mathbf{2 0 0})$ & 253 & 118 \\
\hline BMI $(<25.0)$ & 31.0 & 24.7 \\
\hline Weight (lbs.) & 210 & 167 \\
\hline Waistline (inch) & 44 & 34 \\
\hline
\end{tabular}

Figure I Comparison of Medical Conditions (2010 vs. 2017). 


\section{Methods}

The author, who is also the patient, checks his body weight twice a day in the morning and at bedtime. Using the finger-piercing method, he measures his fasting plasma glucose (FPG) in the early morning before starting his meals and activities. He also measures his postprandial plasma glucose (PPG) three times a day, approximately two hours after the first-bite of each meal. He checks his blood pressure (BP) at least once a day, preferably in the early morning, but sometimes multiple measurements as needed. Every three months, he goes to the same hospital to analyze his lipid conditions. Therefore, his lipid data amount is only around $1 \%$ of his other health conditions such as weight, glucose, and BP.

Using customized artificial intelligence (AI) software on his iPhone, he collected various detailed lifestyle data such as medication, salt intake, stress, sleep, illness, water, food and meal quantity, carbs/ sugar intake, exercise, weather, etc. Finally, he has utilized these big data to conduct many useful correlation analyses among weight, chronic diseases, and lifestyle factors.

From 2015 to 2017, based on his metabolism model, he developed four prediction models, including weight, fasting plasma glucose (FPG), postprandial plasma glucose (PPG), and hemoglobin A1C (A1C). All of these prediction models have reached to $95 \%$ to $99 \%$ data accuracy over a period of approximately four years $(\sim 1,500$ days). Utilizing these prediction models, he could successfully reduce both of his weight and blood sugar. His BMI scores were classified as obese at first, then overweight, and finally to normal range. As a result, his diabetes is under control and both of his hypertension and hyperlipidemia conditions are no longer health issues. During the spring of 2018, he further extended his T2D research into the study of Risk probability of having heart attack and stroke due to chronic diseases

During a period of 2,391 days (1/1/2012 - 7/20/2018) with $\sim 30,000$ collected raw data, which are directly related to this paper, the author further extracted other relevant information out from this big data pool in order to process them according to his designed process flow. He finally categorized them according to an annualized format for easier viewing of data trends from year to year (Figure 6).

Although the author suffered three separate cardiac episodes from 2001 to 2006, he did not link his health risks to his chronic diseases due to his ignorance. Therefore, he hardly kept any data during that decade except for keeping a few significant health records. In 2010, he got his wake-up call from the strong possibility of having dialysis when he received the following lab results: albumin/ creatinine ratio $116.4 \mathrm{mg} / \mathrm{g}, \mathrm{A} 1 \mathrm{C} 10 \%$ and triglycerides $1161 \mathrm{mg} / \mathrm{dL}$. He then immediately ceased all of his ongoing technology business activities, moved into a stress-free retirement community away from Silicon Valley (one of his stressors), and also launched his own T2D research. However, to start this effort successfully, he dedicated his time to study six chronic diseases first in 2010 and throughout 2011. Therefore, after 2012, he started to collect his complete set of health data, including both medical conditions $(\sim 10 \%)$ and lifestyle details $(\sim 90 \%)$. Within this total set of approximately 1.5 million data, there are about 300,000 raw data collected by the computer and other 1 million + data from information processing, which have been saved in a cloud server for other future studies. It should be noted that both $\mathrm{A} 1 \mathrm{C}$ and lipid data were only available from his quarterly lab tests, not on a daily basis. However, the author has developed a predicted daily $\mathrm{A} 1 \mathrm{C}$ that has reached to $95 \%$ accuracy since he added in an uplifted $3 \%$ to $8 \%$ of "safety margin" in order to provide an early warning to himself. Without this build-in safety margin, his predicted A1C data would reach to $99 \%$ accuracy. ${ }^{1-4}$

Unlike the incomplete data collected in 2010, the data integrity after 2012 is accurate, valid, and complete.

\section{Results}

During the period of $1 / 1 / 2012-7 / 20 / 2018$, his medical condition changes and major lifestyle modifications are shown in Figures 2-6. The summarized highlights of results are listed:

Weight: $-49 / 18 \mathrm{lbs}$. (from 220lbs. in 2000, then 189lbs. in 2012 to 171lbs. in 2018). Period 2012-2014 (3 years) has the most reduction.

1. BMI: $-7.1 / 2.6$ (from 32.1 in 2000 , then 27.6 in 2012 to 25.0 in 2018)

2. Waistline: $-12 / 10$ inches (from 44 " in 2000 , then 42 " in 2012 to 32 " in 2018)

Period of 2014-2018 (5 years) has the most reduction.

3. Food \& Meal Quantity: -28\% (over 7 years) from his normal portion of food (from $112 \%$ in 2012 to $84 \%$ in 2018)

4. Daily Walking: $+10,412$ steps in 5 years (from 7,564 in 2013 to 17,976 in 2018)

Period of 2016-2018 has the maximum exercise amount.

5. Post-Meal Walking: $+2,599$ steps in 4 years (from 1,900 in 2015 to 4,499 in 2018)

Period of 2016-2018 has the maximum exercise amount.

6. Daily Glucose: $-163 / 43 \mathrm{mg} / \mathrm{dL}$ (from 280.0 in 2000 , then 160.0 in 2010 to 117.0 in 2018). Period of 2016-2018 has the lowest glucose values.

7. A1C: $-3.3 \%$ (from $10.0 \%$ in 2010 to $6.7 \%$ in 2018 )

Period of 2016-2018 has the lowest values (after discontinuing diabetes medication).

8. BP (Metabolism Index 3): - 26\% (annual average SBP/DBP dropped from $127 / 85$ to $96 / 64$ ).

9. Risk of heart attack or stroke: - $43 \%$ (from $74 \%$ in 2000 to $31 \%$ in 2018, with 3 cardiac episodes during 2001-2006 and none afterwards)

Based on his research results published in the past (see references), a few key conclusions are described as follows:

a. Main causes of his weight loss are reduced by food quantity consumption and increased daily walking steps.

b. It took 3 years to reduce his weight and 5 years to reduce his waistline.

c. Based on his annualized data, he was obese (BMI $>30)$ prior to 2010 and overweight (BMI between 25 and 30) during 2012-2017. His weight has been less than 170lbs. (BMI is normal, around 24.7) in 2018.

d. His reduced FPG below $120 \mathrm{mg} / \mathrm{dL}$ is driven by his weight 
reduction. The combination of his carbs/sugar intake around 14.3grams and post-meal walking around 4,000 steps has caused his PPG to go below $120 \mathrm{mg} / \mathrm{dL}$ as well.

e. His lab-tested A1C value was reduced from 10\% in 2010 (average daily glucose above $200 \mathrm{mg} / \mathrm{dL}$ ) to $6.7 \%$ in 2018 (average daily glucose around $117 \mathrm{mg} / \mathrm{dL}$ ). It should be noted here that he has discontinued all of his diabetes medication for almost 3 years, since $12 / 8 / 2015$

f. His risk probability of having heart attack or stroke was $74 \%$ in 2000. During the period of 2001 through 2006, he suffered three cardiac episodes and none afterwards. By 2017, the same risk factor has reduced to $26.4 \%$ (comparable with Framingham Study of $26.7 \%$ risk in 2017 ).

g. He was diagnosed with hyperlipidemia in 1992, before his diagnosis of T2D in 1997. He noticed the effects of his hypertension condition in 2013 - 2015. However, after 2015, both of his hyperlipidemia and hypertension are no longer threats to his health.

h. He suffered complications from T2D such as renal dysfunction, infections from his bladder, foot ulcer, eye issues, and so forth. To date, all of these problems seem to be under control.

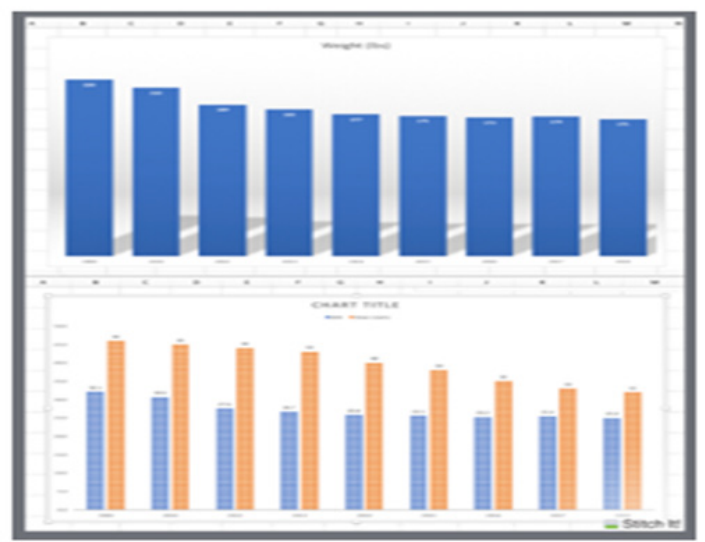

Figure 2 Weight, BMI,Waistline (2000, 20I0, 20I2-20I8).

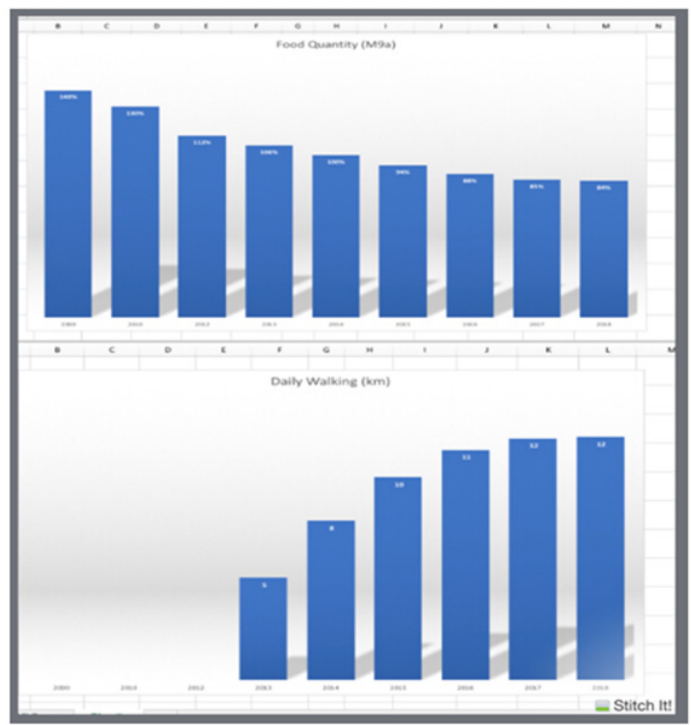

Figure 3 Comparison of Food Quantity \& Walking.

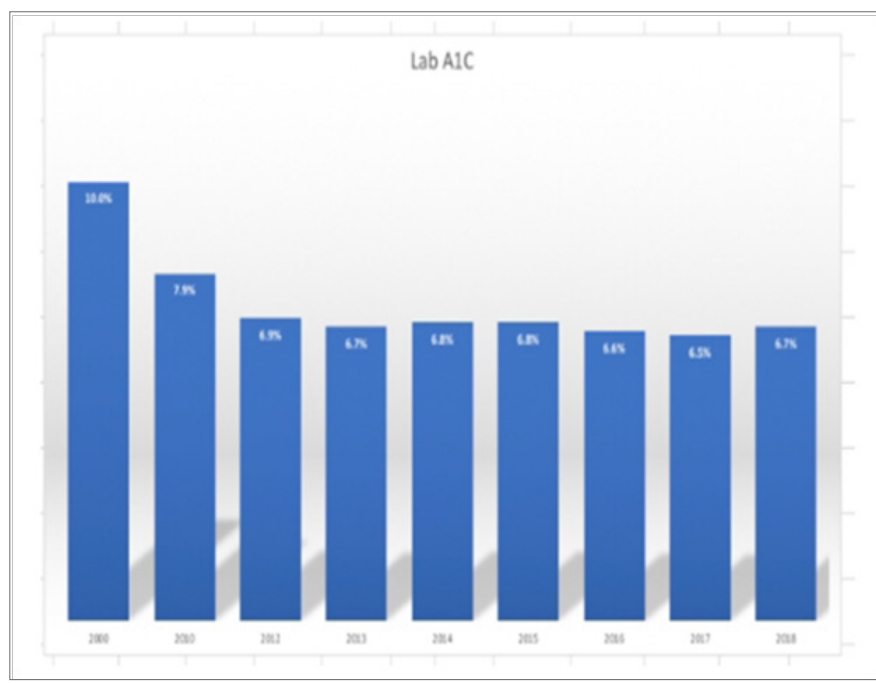

Figure 4 Comparison of Lab-tested A ICValues (2010 - 20I8).

\begin{tabular}{c|c|c|c|c|}
\hline Year & Probability & Probability & Probability & Year \\
\hline & MI & Medical & Lifestyle & Averaged \\
\hline $\mathbf{2 0 0 0}$ & & $\mathbf{7 4} \%$ & $\mathbf{8 3} \%$ & $\mathbf{7 9} \%$ \\
\hline & & & & \\
\hline $\mathbf{2 0 1 2}$ & $\mathbf{7 4} \%$ & $\mathbf{6 2} \%$ & $\mathbf{7 0} \%$ & $\mathbf{6 9} \%$ \\
\hline $\mathbf{2 0 1 3}$ & $\mathbf{7 7} \%$ & $\mathbf{3 8} \%$ & $66 \%$ & $\mathbf{6 0} \%$ \\
\hline $\mathbf{2 0 1 4}$ & $\mathbf{5 9} \%$ & $\mathbf{4 2} \%$ & $\mathbf{5 4} \%$ & $\mathbf{5 1} \%$ \\
\hline $\mathbf{2 0 1 5}$ & $\mathbf{4 3} \%$ & $\mathbf{3 9} \%$ & $44 \%$ & $\mathbf{4 2} \%$ \\
\hline $\mathbf{2 0 1 6}$ & $36 \%$ & $31 \%$ & $38 \%$ & $\mathbf{3 5} \%$ \\
\hline $\mathbf{2 0 1 7}$ & $34 \%$ & $\mathbf{2 6} \%$ & $33 \%$ & $\mathbf{3 1} \%$ \\
\hline $\mathbf{2 0 1 8}$ & $33 \%$ & $\mathbf{3 1} \%$ & $33 \%$ & $\mathbf{3 2} \%$ \\
\hline & & & & \\
\hline $\mathbf{7 - Y e a r s}$ & $\mathbf{5 2} \%$ & $\mathbf{3 4} \%$ & $34 \%$ & $\mathbf{4 0} \%$ \\
\hline
\end{tabular}

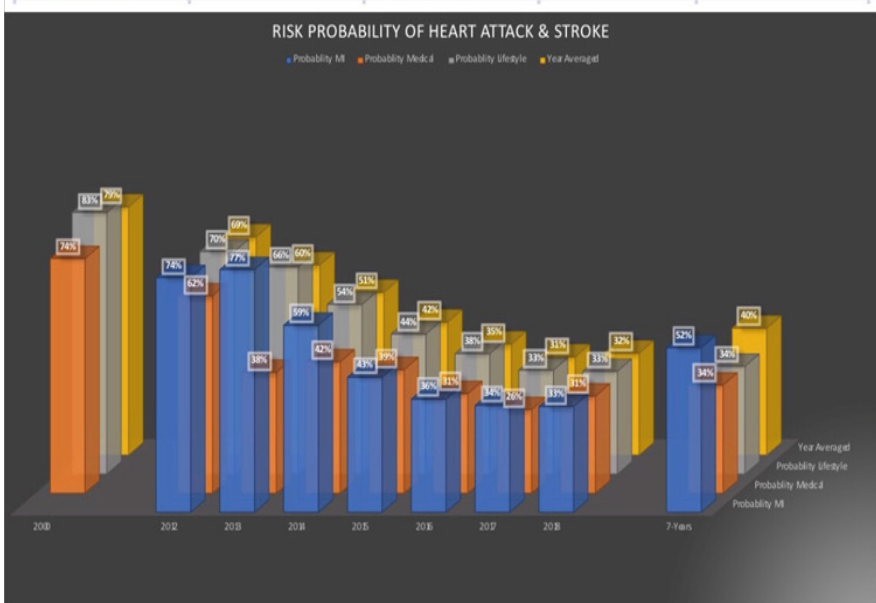

Figure 5 Risk Probability of Heart Attack \& Stroke (20I0, 20I2-20I8) 


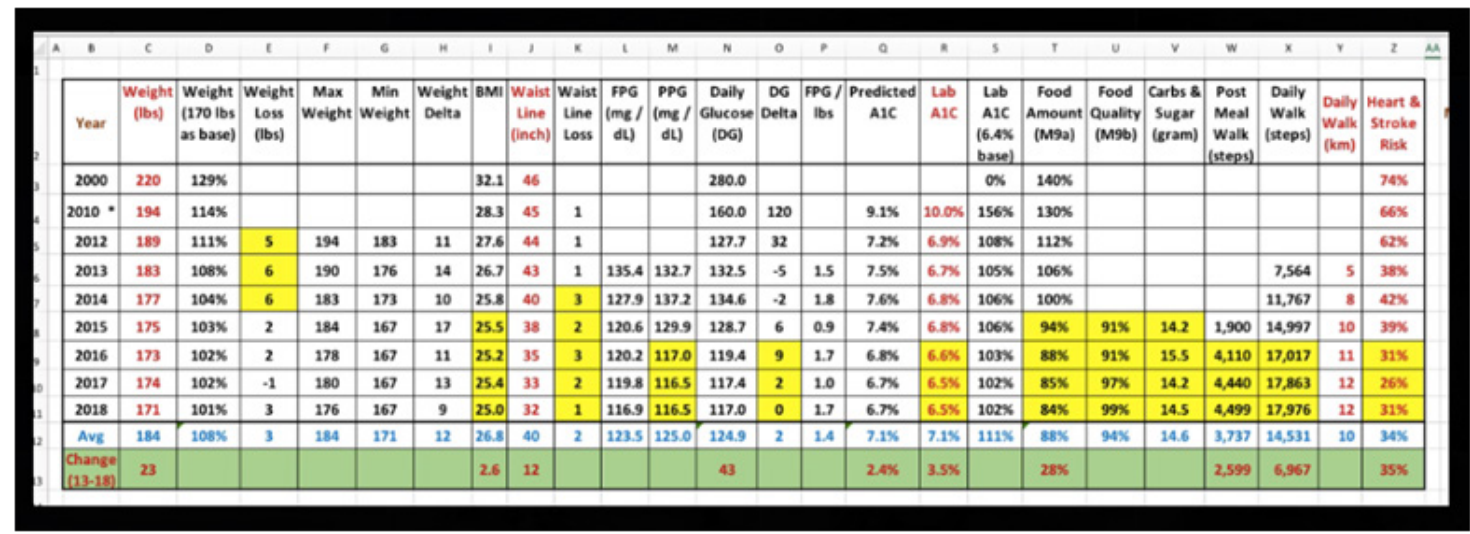

Figure 6 Annualized Data Comparison (2000, 2010, 20I2-20I8).

\section{Conclusion}

This report is about one patient (the author) over a 20-year period. He has collected and processed approximately 1.5 million data, particularly during the period of 2012-2018. He has spent 20,000 hours to conduct his research regarding chronic diseases and his own health. This dynamic big data analytics using tools, such as advanced mathematics, physics, signal processing, optical physics, engineering modeling, machine learning and AI, should be equally effective for analyzing other patient's conditions. Although some data may be slightly different, the results and conclusions should be applicable to other T2D cases. In this article, there are no new breakthrough findings about medicine, but the author is able to provide quantitative proof to support those existing knowledge in modern medical community.

\section{Limitations of research}

This article is based on data of metabolic conditions and lifestyle details collected from one T2D patient (himself). It does not cover genetic conditions and lifestyle details of other diabetes patients. However, the author's research approach is based on his solid interdisciplinary academic background and successful multiple industrial experiences. His academic background and working experience have prepared him to conduct his diabetes research with the following thorough process and carefully chosen steps:

Observing and identifying a system's basic characters like a pure physicist;

1) Developing related but rigorous mathematical equations like a mathematician;

2) Applying suitable engineering models and useful statistical models to address the real-world challenges;

3) Using modern computer science tools and sophisticated AI techniques to aid in problem solving.

Nevertheless, his conclusions and findings should be re-verified and proceed with caution when applying to other patients who are under different metabolic conditions or lifestyles.

\section{Acknowledgements}

First and foremost, the author wishes to express his sincere appreciation to a very important person in his life, Professor Norman Jones at MIT and University of Liverpool. Not only did he give him the opportunity to study for his $\mathrm{PhD}$ at MIT, but he also trained him extensively on how to solve difficult problems and conduct any basic scientific research with a big vision, pure heart, and integrity.

The author would also like to thank Professor James Andrews at the University of Iowa. He helped and supported him tremendously when he first came to the United States. His encouragement assisted him to build his solid engineering and computer science foundation. $\mathrm{He}$ is forever grateful to his mentor, who has a kind heart and guided him during his undergraduate and master's degree work.

\section{Conflicts of interest}

The author declares there are no conflicts of interest.

\section{References}

1. Hsu, Gerald C. Using Math-Physical Medicine to Control T2D via Metabolism Monitoring and Glucose Predictions. Journal of Endocrinology and Diabetes. 2018;1(1):1-6.

2. Hsu, Gerald C. Using Math-Physical Medicine to Analyze Metabolism and Improve Health Conditions. Video presented at the meeting of the 3rd International Conference on Endocrinology and Metabolic Syndrome 2018, Amsterdam, Netherlands.

3. Hsu, Gerald C. Using Signal Processing Techniques to Predict PPG for T2D. International Journal of Diabetes \& Metabolic Disorders. 2018;3(2):1-3.

4. Hsu, Gerald C. Using Math-Physical Medicine and Artificial Intelligence Technology to Manage Lifestyle and Control Metabolic Conditions of T2D. International Journal of Diabetes \& Its Complications. 2018;2(3):17. 